\title{
Increased Expression of the Drosophila Vesicular Glutamate Transporter Leads to Excess Glutamate Release and a Compensatory Decrease in Quantal Content
}

\author{
Richard W. Daniels, ${ }^{1 \star}$ Catherine A. Collins, ${ }^{1 \star}$ Maria V. Gelfand, ${ }^{1}$ Jaime Dant, ${ }^{1}$ Elizabeth S. Brooks, ${ }^{2}$ David E. Krantz, ${ }^{2}$ \\ and Aaron DiAntonio ${ }^{1}$ \\ ${ }^{1}$ Department of Molecular Biology and Pharmacology, Washington University School of Medicine, St. Louis, Missouri 63110, and ²Department of \\ Psychiatry and Biobehavioral Sciences, The David Geffen School of Medicine at University of California Los Angeles, Los Angeles, California 90095
}

\begin{abstract}
Quantal size is a fundamental parameter controlling the strength of synaptic transmission. The transmitter content of synaptic vesicles is one mechanism that can affect the physiological response to the release of a single vesicle. At glutamatergic synapses, vesicular glutamate transporters (VGLUTs) are responsible for filling synaptic vesicles with glutamate. To investigate how VGLUT expression can regulate synaptic strength in vivo, we have identified the Drosophila vesicular glutamate transporter, which we name DVGLUT. DVGLUT mRNA is expressed in glutamatergic motoneurons and a large number of interneurons in the Drosophila CNS. DVGLUT protein resides on synaptic vesicles and localizes to the presynaptic terminals of all known glutamatergic neuromuscular junctions as well as to synapses throughout the CNS neuropil. Increasing the expression of DVGLUT in motoneurons leads to an increase in quantal size that is accompanied by an increase in synaptic vesicle volume. At synapses confronted with increased glutamate release from each vesicle, there is a compensatory decrease in the number of synaptic vesicles released that maintains normal levels of synaptic excitation. These results demonstrate that (1) expression of DVGLUT determines the size and glutamate content of synaptic vesicles and (2) homeostatic mechanisms exist to attenuate the excitatory effects of excess glutamate release.
\end{abstract}

Key words: synaptic vesicle; quantal size; vesicular glutamate transporter; Drosophila; glutamate; synaptic transmission

\section{Introduction}

Synaptic strength is determined both by quantal content (QC), the number of synaptic vesicles (SVs) released by the presynaptic terminal, and by quantal size, the postsynaptic response to the transmitter released from each vesicle. The regulation of postsynaptic receptor activity is the best-studied mechanism for controlling quantal size (Malinow and Malenka, 2002); however, the time course and concentration of transmitter in the synaptic cleft also affect the postsynaptic response (Choi et al., 2000; Renger et al., 2001; Liu, 2003; Pawlu et al., 2004). Therefore, the transmitter content of synaptic vesicles represents another potential determinant of quantal size. Because vesicular neurotransmitter transporters load vesicles with transmitter, their expression and activity may determine the transmitter content of synaptic vesicles.

\footnotetext{
Received July 22, 2004; revised 0ct. 12, 2004; accepted 0ct. 12, 2004.

This work was supported by grants from the National Institute of Neurological Disorders and Stroke (NS043171), the Keck Distinguished Young Scholars Program, and the McKnight Scholars Award (A.D.), a National Institutes of Health training grant (GM08151) (R.W.D.), the Damon Runyon Foundation (C.A.C.), a Howard Hughes Medical Institute summer fellowship (M.V.G.), and grants from the National Institute of Mental Health (MH01709), the National Institute of Diabetes and Digestive and Kidney Diseases (DK60857), the National Institute of Environmental Health Sciences (ES-02-03), and the Edward Mallinckrodt Jr and EJLB Foundations (D.E.K.). We thank Regis Kelly and Vivian Budnik for reagents and Sylvia Johnson and Scott Portman for technical assistance.

DOI:10.1523/JNEUROSCI.3001-04.2004

${ }^{*}$ R.W.D. and C.A.C. contributed equally to this work.

Correspondence should be addressed to Dr. Aaron DiAntonio, Department of Molecular Biology and Pharmacology, Campus Box 8103, 660 South Euclid, Washington University School of Medicine, St. Louis, M0 63110. E-mail: diantonio@wustl.edu.

Copyright $\odot 2004$ Society for Neuroscience $\quad$ 0270-6474/04/2410466-09\$15.00/0
}

Three families of vesicular neurotransmitter transporters have been identified: the vesicular transporters for monoamines (VMATs) and acetylcholine (VAChT) (Erickson et al., 1992, 1994; Liu et al., 1992; Roghani et al., 1994), the vesicular transporter for GABA and glycine (VGAT or VIAAT) (McIntire et al., 1997), and the three recently isolated vesicular glutamate transporters (VGLUTs 1-3) (Bellocchio et al., 2000; Takamori et al., 2000; Fremeau et al., 2002). Expression of these transporters can regulate the transmitter content of vesicles and quantal size; overexpression of VMAT (Colliver et al., 2000; Pothos et al., 2000), VAChT (Song et al., 1997), and VGLUT1 (Wojcik et al., 2004) in various cultured cells increases quantal size. For monoamines, this increase in quantal size is accompanied by an increase in vesicular volume (Colliver et al., 2000; Gong et al., 2003). The relationship among transporter expression, transmitter content, and vesicle size has not been investigated for classical fast transmitters such as glutamate.

VGLUTs load synaptic vesicles with glutamate. The deletion of VGLUT1 in the mouse leads to a dramatic impairment of glutamatergic transmission (Fremeau et al., 2004; Wojcik et al., 2004). Conversely, overexpression of VGLUT1 in cultured glutamatergic neurons increases quantal size at autaptic synapses (Wojcik et al., 2004); however, the functional consequences of excess glutamate for the efficacy of an intact synapse are unknown.

To explore how the expression of VGLUT within its normal synaptic milieu regulates synaptic strength, we identified and 
characterized the Drosophila VGLUT homolog, DVGLUT. DVGLUT localizes to synaptic vesicles, is expressed in glutamatergic motoneurons and interneurons, and is present at the synaptic terminals of all identified glutamatergic neuromuscular junctions (NMJs). Overexpression of DVGLUT in motoneurons increases quantal size by shifting the entire population of miniature excitatory junctional potentials (mEJPs) to larger amplitudes. This physiological change is accompanied by a morphological change: synaptic vesicles are larger. This suggests that DVGLUT expression can regulate the total glutamate content of a vesicle but that the concentration of glutamate stays roughly constant. Finally, we investigated the consequences of this excess glutamate release for the function of the intact NMJ. Despite the increased postsynaptic response to single vesicles, the response to the evoked release of vesicles was unchanged because of a compensatory downregulation of presynaptic vesicle release. Hence, homeostatic mechanisms exist at the Drosophila NMJ to attenuate the excitatory effects of excess glutamate release.

\section{Materials and Methods}

Genetics. cDNAs for CG9887 (RH57669 and RH74545) and for CG4288 (AT07766 and AT14282) were obtained from the Berkeley Drosophila Genome Project expressed sequence tag collection. The two cDNAs for CG9887 were sequenced to completion and are identical. Both demonstrated that the annotated predicted protein product for CG9887 is missing a $207 \mathrm{bp}$ exon that was predicted to be part of the final intron. This added exon encodes an additional transmembrane domain that is also present in vertebrate VGLUTs.

To generate the upstream activating sequence (UAS)-DVGLUT line, the CG9887 cDNA was synthesized by PCR using a 5' PCR oligo GGGAATTCATGAAGGGTCTGACGGCGTT that introduced an EcoRI site (underlined) and $3^{\prime}$ oligo CGGTACCTTACTGCTGCTGGTATCCCT that introduced a $K p n \mathrm{I}$ site (underlined), sequenced, cloned into the pUAST vector (Brand and Perrimon, 1993), and transformed into embryos using standard techniques.

Drosophila were cultured using standard techniques. Crosses were kept at $25^{\circ} \mathrm{C}$ on standard Drosophila medium. The BG380 Gal4 line expresses in motoneurons and was a kind gift of Vivian Budnik (Budnik et al., 1996). In all experiments, control larvae (referred to as wild type) were BG380 Gal4 virgins out-crossed to Canton-S (CS) males.

In situ hybridization. Specific antisense probes were synthesized and hybridized to a $24 \mathrm{hr}$ collection of dechorionated embryos using conventional methods described previously (Tautz et al., 1989). Briefly, we transcribed RNA probe from linearized, phenol-chloroform-extracted DNA from clone AT07766 for CG4288 and clone RH57669 for CG9887.

Biochemistry. Glycerol gradient fractionations were performed as described (van de Goor et al., 1995) with minor modifications. Approximately $100 \mathrm{mg}$ of frozen fly heads were crushed over dry ice using a mortar and pestle and then homogenized in an ice bath in $0.5 \mathrm{ml}$ of $1 \mathrm{mM}$ EGTA, $0.1 \mathrm{~mm} \mathrm{MgCl}_{2}, 10 \mathrm{~mm}$ HEPES, $\mathrm{pH} 7.4,1 \mathrm{mg} / \mathrm{ml}$ pepstatin, $2 \mathrm{mg} / \mathrm{ml}$ leupeptin, $20 \mathrm{mg} / \mathrm{ml}$ PMSF using eight strokes of a motorized Teflon pestle on glass (Wheaton) at $900 \mathrm{rpm}$. After debris was sedimented at $1000 \times g$ for $2 \mathrm{~min}$ at $4^{\circ} \mathrm{C}$ in a Microfuge, the homogenate was loaded onto a 5-25\% v/v glycerol gradient containing (in $\mathrm{mm}$ ): $150 \mathrm{NaCl}$, 1 EGTA, $0.1 \mathrm{MgCl}_{2}, 10 \mathrm{HEPES}, \mathrm{pH}$ 7.4. The gradient was generated using a BioComp Gradient Master (Fredericton, New Brunswick, Canada) followed by the addition of a $50 \%$ sucrose cushion at the bottom of the tube to capture rapidly sedimenting membranes. After centrifugation at 40.4 $\mathrm{K} \mathrm{rpm}$ in an SW41 rotor (Beckman) for $75 \mathrm{~min}$ at $4^{\circ} \mathrm{C}$, fractions were recovered from the bottom of the tube using a Beckman Fraction Recovery System. Samples from each fraction were probed on Western blots using primary antibodies to DVGLUT $(1: 10,000)$ and Drosophila synaptobrevin (1:2000), generously provided by Regis Kelly (University of California San Francisco, San Francisco, CA), and late bloomer (lbm) (1:100) (Kopczynski et al., 1996) followed by the appropriate HRPconjugated secondary antibody (Amersham Biosciences, Arlington
Heights, IL) and a chemiluminescent substrate (SuperSignal West Pico, Pierce, Rockford, IL). Digital images were quantified using NIH Image.

Electrophysiology. Electrophysiological recordings were performed as described previously (Marrus and DiAntonio, 2004; Marrus et al., 2004). Briefly, wandering third-instar female larvae were dissected in ice-cold Stewart's HL-3 solution (Stewart et al., 1994) with no added calcium. HL-3 solution contained (in $\mathrm{mm}$ ): $70 \mathrm{NaCl}, 5 \mathrm{KCl}, 20 \mathrm{MgCl}_{2}, 10$ $\mathrm{NaHCO}_{3}, 5$ trehalose, 115 sucrose, and 5 HEPES, pH adjusted to $7.2 ; 1 \mathrm{M}$ $\mathrm{CaCl}_{2}$ was added to achieve the desired $\mathrm{Ca}^{2+}$ concentration. Intracellular recordings were made from muscle 6 , segments $\mathrm{A} 3$ and A4, with sharp borosilicate electrodes with resistance of $17-20 \mathrm{M} \Omega$ when filled with $3 \mathrm{M}$ $\mathrm{KCl}$. Signals were acquired using an AxoClamp-2B amplifier (Axon Instruments, Foster City, CA) in bridge mode and then filtered at $1 \mathrm{kHz}$ and amplified using a Model 410 amplifier (Brownlee Precision, San Jose, CA). Data were digitized using a Digidata 1320A board and stored on a computer using pClamp 9.0 software. Cells were selected for data analysis if input resistance was $\geq 5 \mathrm{M} \Omega$ and membrane potential was less than $-60 \mathrm{mV}$. All recordings were made at room temperature.

Spontaneous mEJPs were recorded in nominally $\mathrm{Ca}^{2+}$-free HL-3 solution with $1 \mu \mathrm{M}$ TTX added to prevent action potentials. The largest mEJP recorded from the DVGLUT-overexpressing larvae was $32 \mathrm{mV}$. After recording from each cell, the nerve was stimulated 10 times to ensure that no events could be evoked. At least 60 consecutive events were measured per cell using MiniAnal (Synaptosoft, Decatur, GA) and averaged to determine the mean $\mathrm{mEJP}$. Events with a slow rise time course were rejected as artifacts from neighboring electrically coupled muscle cells. There is no statistically significant difference in the resting potential from wild-type and DVGLUT-overexpressing larvae (BG380 Gal4/+ $V_{\mathrm{m}}=$ $68.1 \pm 1.4 \mathrm{mV}$; BG380 Gal4/+; UAS-DVGLUT $1 /+V_{\mathrm{m}}=69.5 \pm 2.2 \mathrm{mV}$; BG380 Gal4/+; UAS-DVGLUT $2 /+V_{\mathrm{m}}=69.5 \pm 2.2 \mathrm{mV}$ ). Failure analysis was performed in HL-3 solution containing $0.23 \mathrm{mM} \mathrm{CaCl}_{2}$, a level at which approximately half of the stimulations elicited responses in the muscle in larvae overexpressing DVGLUT. Failures were counted by hand in both genotypes because of large fluctuations in the baseline voltage caused by large electrically coupled events in neighboring muscles in overexpressing larvae. Statistical analysis was performed and graphs were generated in Origin 7.0 (Origin Lab, Northampton, MA).

Antibody staining. Wandering third-instar larvae were dissected in icecold PBS and fixed in Bouin's fixative (1:5:15 ratio of acetic acid/formalin/picric acid) for $5 \mathrm{~min}$. For anti-cysteine string protein (CSP) staining, larvae were fixed for $45 \mathrm{~min}$ in Bouin's fixative. Larvae were then washed in PBS containing $0.1 \%$ Triton-X (PBX) and blocked for $1 \mathrm{hr}$ at room temperature in 5\% normal goat serum in PBX. Primary antibody was diluted in PBS and incubated overnight at $4^{\circ} \mathrm{C}$ or at room temperature for $1 \mathrm{hr}$. Secondary antibodies were used at 1:2000 for $1 \mathrm{hr}$ at room temperature. After staining, larvae were equilibrated in 70\% glycerol in PBS and mounted with VectaShield (Vector Laboratories, Burlingame, CA).

Antibodies. The rabbit anti-DVGLUT antibody was raised against a C-terminal peptide (-CQMPSYDPQGYQQQ) by Zymed Laboratories (San Francisco, CA) and purified on an affinity column. Purified antibody was used at 1:10,000-1:25,000. This antisera recognizes a single band of approximately the expected size for CG9887 on an immunoblot and detects the transgenic overexpression of CG9887. Mouse anti-CSP (ab49) was kindly provided by Konrad Zinsmaier (University of Arizona, Tucson, AZ) and used at 1:50. Cy3-conjugated goat anti-HRP (Jackson ImmunoResearch, West Grove, PA) was used at 1:1000. Differences in synaptic DVGLUT level were assessed using an Alexa-488-coupled DVGLUT antibody at 1:1000.

Comparison of protein levels. Larvae of different genotypes were dissected and stained together to ensure uniform processing. Confocal micrographs were taken with a Nikon $\mathrm{C} 1$ confocal microscope of the synapse on muscle 4 of segments A3 and A4 using a $60 \times$ oil objective (numerical aperture $=1.40$ ) with a pixel size of $200 \mathrm{~nm}$. Gain was set to just below saturation on the sample with the highest staining intensity. Complete Z-stacks through the synapse were flattened into a maximum projection and saved for analysis. Analysis of staining was performed using MetaMorph software (Universal Imaging, West Chester, PA). Briefly, images were thresholded using the color threshold function, the 
synaptic region was defined in the HRP channel, and the intensity of DVGLUT within that region was measured.

Electron microscopy. Larvae were dissected in freshly prepared Sylgardlined Petri dishes with new insect pins in nominally $\mathrm{Ca}^{2+}$-free $\mathrm{HL}-3$ solution and fixed for $1 \mathrm{hr}$ at $4^{\circ} \mathrm{C}$ in electron microscopy grade $2.0 \%$ paraformaldehyde, $2.5 \%$ glutaraldehyde, and $1 \%$ tannic acid in $0.1 \mathrm{M}$ cacodylic acid buffer, $\mathrm{pH} 7.2$ (CB). Larvae were then unpinned and transferred into $1 \% \mathrm{OsO}_{4}$ in $\mathrm{CB}$ for $1 \mathrm{hr}$ at room temperature and stained en bloc with $1 \%$ uranyl acetate in $\mathrm{H}_{2} \mathrm{O}$. Tissue was dehydrated in a progressive concentration series of ethanol and propylene oxide and embedded in Epon resin (Electron Microscopy Sciences, Hatfield, PA). Blocks were sectioned in an RMC Ultra microtome at $\sim 70 \mathrm{~nm}$ thickness with a Delaware diamond knife (Wilmington, DE) and poststained for $1 \mathrm{hr}$ in Reynolds lead citrate and uranyl acetate. Sections were viewed and micrographs taken on a JOEL 100C transmission electron microscope.

Image analysis. Electron micrographs were taken from a total of 201 active zones from muscles 7 and 6 from segments A2-A4 from two larvae of each genotype. A total of 35 boutons from four nerves were used in overexpressors, and 31 boutons from six nerves were used in wild type. Each active zone was cropped in Photoshop and randomly coded. The diameters of vesicles near active zones were measured blind to genotype using MetaMorph software. Seventeen hundred vesicle diameters from each genotype were used for the histograms shown in Figure 5.

\section{Results}

\section{A Drosophila homolog of vertebrate VGLUT}

We searched the Drosophila genome for sequences that could encode an ortholog of the vertebrate VGLUT genes. The closest Drosophila relative is an uncharacterized predicted gene called CG9887, which shows 41\% identity with human VGLUT1, 40\% identity with both mouse and rat VGLUT2, and 39\% identity with the Caenorhabditis elegans VGLUT1 homolog EAT-4 (Fig. 1a). In addition to having a well conserved sequence, CG9887 also has a very similar hydrophobicity profile to the vertebrate VGLUTs (Fig. 1b). Both CG9887 and the VGLUTs are highly hydrophobic, with many predicted membrane-spanning domains. Amino acid identity within this highly hydrophobic region is 53\%. After CG9887, the nearest Drosophila homolog to the vertebrate VGLUTs is the uncharacterized gene CG4288. It shows 35\% identity to VGLUT1 and also has a similar hydrophobicity profile (data not shown).

\section{Expression and vesicular localization of DVGLUT}

The Drosophila vesicular glutamate transporter must be expressed in glutamatergic cells and localized to synaptic vesicles at glutamatergic synapses. The Drosophila NMJ is glutamatergic, so we would expect to find mRNA for the bona fide transporter in the cell bodies of motoneurons. In situ hybridization demonstrates that CG9887 mRNA is expressed in a subset of central neurons in embryos (Fig. 2a), including RP motoneurons as well as a group of lateral cells that are likely to be the lateral motoneuron cluster. CG9887 mRNA is expressed at a number of other sites in the embryonic CNS; it is first detected at embryonic stage 13 in a small group of segmentally repeating cells and later in a large number of cells that are likely to be interneurons. In contrast, we were unable to detect expression of CG4288 mRNA in any embryonic neurons (data not shown). Because the expression pattern of CG9887, but not CG4288, is consistent with that expected for the Drosophila VGLUT, we initiated an analysis of the protein encoded by CG9887.

Vesicular glutamate transporters function at the synapse on synaptic vesicles. If CG9887 encodes the Drosophila VGLUT, it should be present at glutamatergic terminals. Drosophila NMJs can be differentiated into type Ib, Is, II, and III based on bouton morphology and muscle target. Type Ib and Is terminals are the a DVGLUT (1) MRGLTAFKEKATGVFGGLKPNMEKFEISQSYHGGHGGYEEMEGGGDEGRG VGLUT1 (1) -

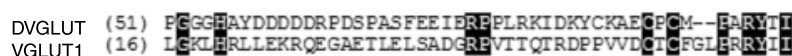
DVGLUT (99) ATYACYTRYT

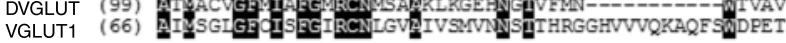

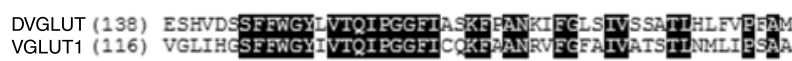

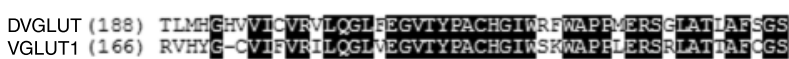

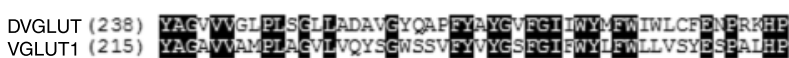

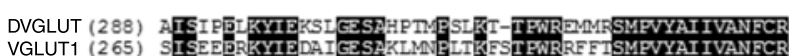

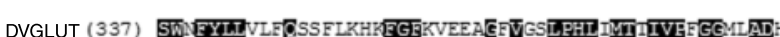

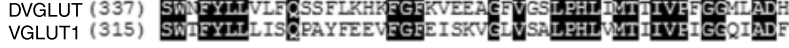

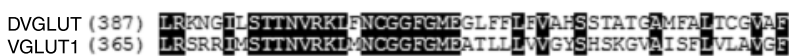
DVGLUT (437) SGFAISGYNWNHLDIAPRYASILWEISNGIGTIAGIIVFYALDGLIOANP VGLUT1 (415) SGFAISG PWVNHLDIAPRYASILNGISNGVGIISGMVCI IVGAMTKHKT

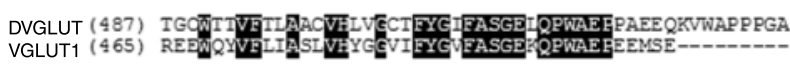
DVGLUT (537) ITNTDPSQRGMIGDMMETSFGAPEYTE CSOMIQSTAISYGATGHVANNP VGLUT1 (506) -----EKCGFG GHDLAG------SDDGEYEDEAE--------DVGLUT (587) FAMASGAPFIAEEDIPFTYGDVINPGQQYGYTQGQMPSYPPQGYQQQ VGLUT1 (530) -------PEGAPPAEPBYGATHSTEQPPRPPPVRDY-------

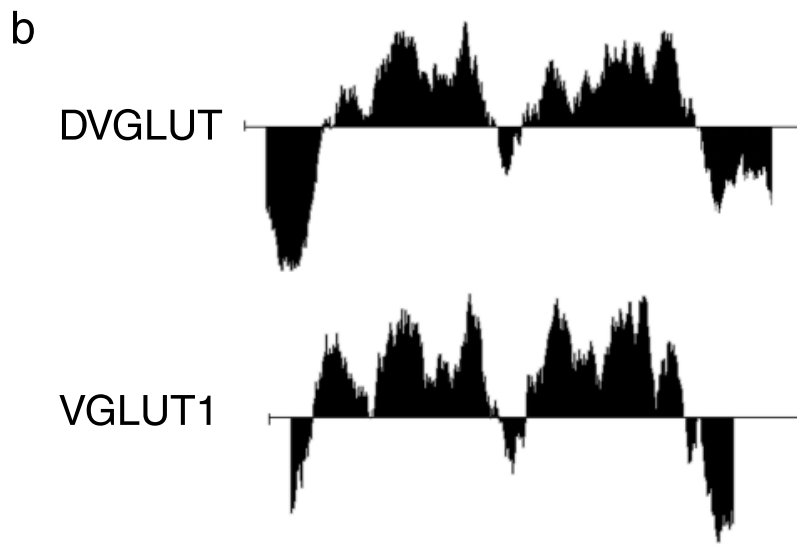

Figure 1. Sequence of DVGLUT. a, Sequence and alignment of DVGLUT (Drosophila CG9887) and human VGLUT1 with identical residues shaded. Overall amino acid identity is $41 \%$. $b$, Hydropathy plots of human VGLUT1 and DVGLUT demonstrate that both proteins have very similar hydrophobicity profiles and are likely to have similar membrane topology.

classic glutamatergic terminals that mediate excitatory transmission, whereas type II terminals contain neuropeptides and are thought to be neuromodulatory, and the type III terminals likely secrete an insulin-like peptide. The type II terminals, however, also contain high levels of glutamate as assayed by an antiglutamate antibody, and ionotropic glutamate receptors cluster opposite not only Ib and Is synapses but also type II synapses (Johansen et al., 1989; Marrus et al., 2004). We generated an antibody to a unique C-terminal peptide from CG9887. This antisera to CG9887 intensely stains all type Ib, Is, and II boutons but not the type III terminal. Additionally, staining for CG9887 shows complete colocalization at type I and II terminals with staining for the CSP, a known synaptic vesicle protein (Fig. 2b) (Zinsmaier et al., 1994).

Although immunocytochemistry is consistent with a vesicular localization for CG9887, we wanted to test directly whether the CG9887 protein is present on synaptic vesicles. We performed biochemical isolation of synaptic vesicles from fly-head extracts using glycerol gradient centrifugation. Because synaptic vesicles are relatively small and uniform in size, they migrate more slowly 

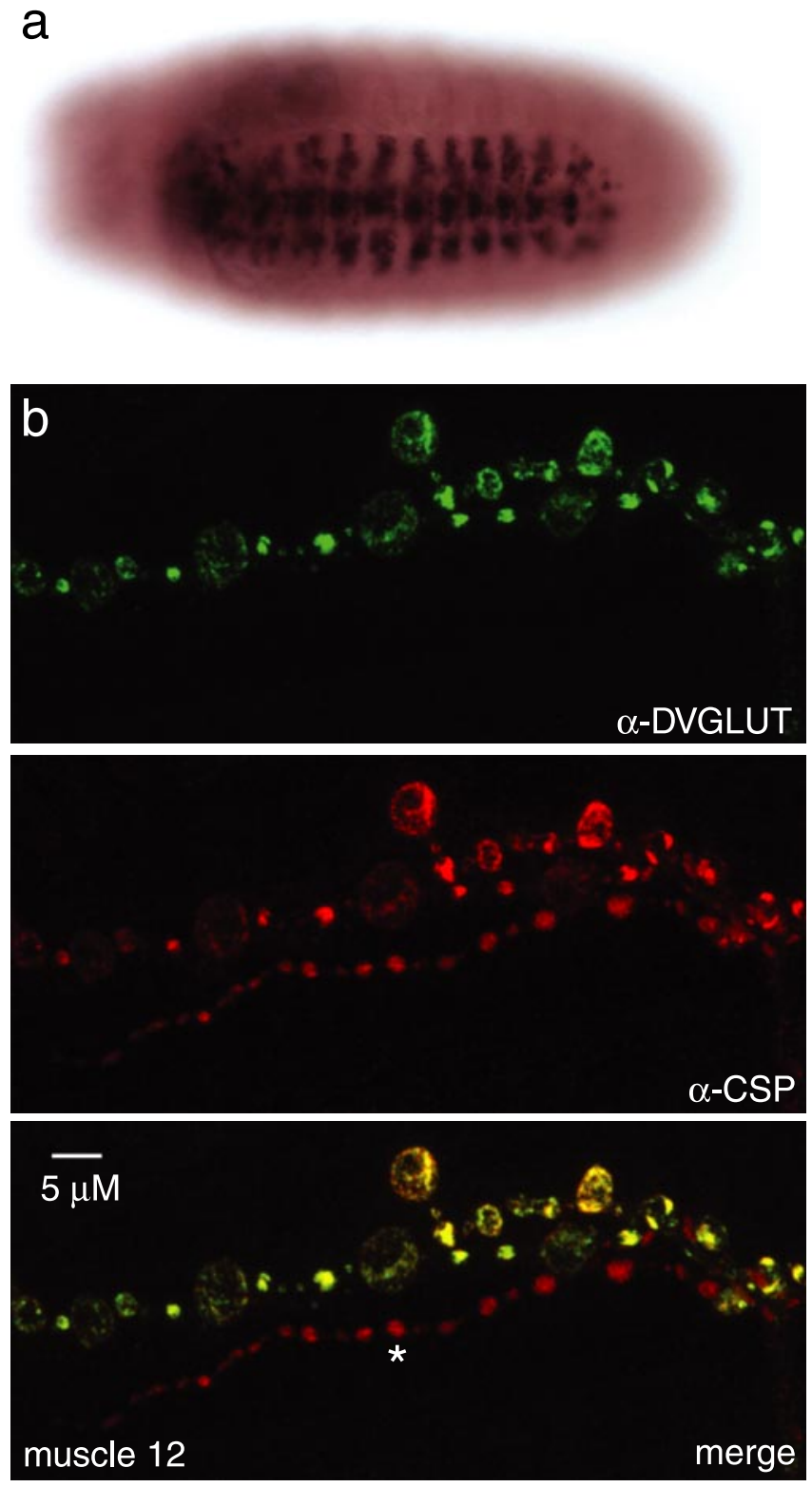

Figure 2. Expression pattern of DVGLUT. a, Embryonic in situ hybridization to DVGLUT transcript demonstrates expression in a subset of cells in the ventral nerve cord, including putative motoneuron cell bodies and interneurons. $b$, Confocal fluorescence microscopy of muscle 12 in a third-instar larva reveals that DVGLUT (green) colocalizes with the synaptic vesicle protein CSP (red) in type lb and Is NMJs. Note in the merged image that DVGLUT is not expressed at the type III input (indicated with an asterisk) onto muscle 12. Scale bar, $5 \mu \mathrm{m}$.

than the plasma membrane and other organelles, which rapidly sediment to the bottom of this gradient, as shown previously for both cultured neuroendocrine cells and Drosophila neurons (Clift-O'Grady et al., 1990; van de Goor et al., 1995). The v-soluble $N$-ethylmaleimide-sensitive factor attachment protein receptor (SNARE) n-synaptobrevin (DiAntonio et al., 1993; van de Goor et al., 1995) cosediments with the CG9887 protein in fractions enriched for synaptic vesicles (Fig. 3), indicating that CG9887 localizes to synaptic vesicles. In contrast, lbm (Kopczynski et al., 1996), a tetraspanin that localizes to the plasma membrane, sediments to the bottom of the gradient. Together, these results demonstrate that CG9887 has high homology to VGLUTs, is expressed in known glutamatergic cells, localizes to synaptic terminals, and is a synaptic vesicle protein. Therefore, we suggest that CG9887 is the Drosophila VGLUT and therefore rename CG9887 as DVGLUT. a

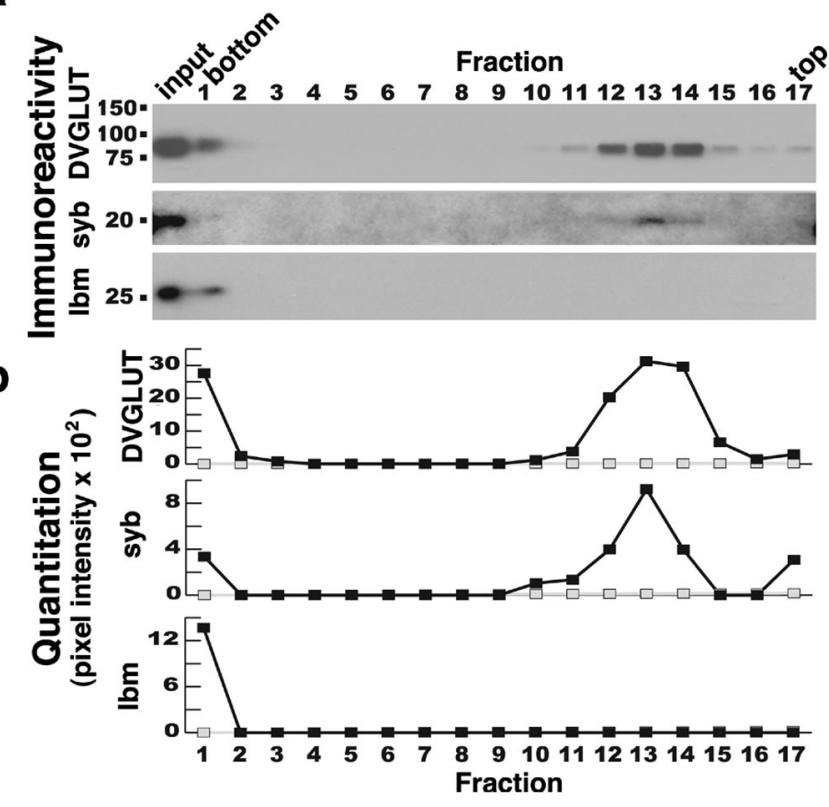

Figure 3. DVGLUT is a synaptic vesicle protein. $a$, DVGLUT cosediments with the synaptic vesicle protein $n$-synaptobrevin after glycerol gradient fractionation. Individual fractions from the gradient were analyzed by immunoblot with antisera against DVGLUT (top), n-synaptobrevin (syb; middle), and Ibm (bottom). b, Quantitation of the immunoblot in $a$ demonstrates that DVGLUT and $n$-synaptobrevin cosediment but lbm does not. Fractions representing the top and bottom of the gradient are indicated. A sample of the homogenate that was loaded onto the gradient was included for comparison (input).

\section{Increased quantal size with DVGLUT overexpression}

It was demonstrated recently that viral overexpression of mouse VGLUT1 in cultured hippocampal neurons leads to an increase in quantal size at autaptic synapses (Wojcik et al., 2004). We wanted to investigate whether the expression of DVGLUT could regulate quantal size in vivo when overexpressed in its normal synaptic environment. To achieve tissue-specific overexpression of DVGLUT, we used the Gal4/UAS transcriptional activation system (Brand and Perrimon, 1993). We generated transgenic flies in which the DVGLUT cDNA is under the control of the yeast $U A S$ promoter ( $U A S-D V G L U T)$ ). To restrict expression to neurons, we crossed these flies to the neuronal Gal4 line BG380 Gal4 (Budnik et al., 1996). This led to a large increase of DVGLUT protein at the synapse; synaptic staining for DVGLUT in larvae overexpressing the DVGLUT transgene was increased approximately threefold compared with controls (Fig. $4 a)(n=16$ NMJs; $\mathrm{p} \ll 0.001)$.

To test the hypothesis that DVGLUT levels can regulate quantal size in vivo, we recorded spontaneous mEJPs from muscle 6 of segments A3 and A4 from control larvae and larvae overexpressing DVGLUT. These mEJPs represent the electrical response in the muscle to the spontaneous fusion of a single synaptic vesicle with the presynaptic membrane. The amplitude of the mEJP depends in part on the activity of the postsynaptic glutamate receptors. Because these receptors are not saturated at this synapse, the amplitude of the mEJP should also depend on the amount of glutamate released from each vesicle (Karunanithi et al., 2002). When DVGLUT is overexpressed presynaptically, we find an increase in the mEJP amplitude, a result that is consistent with an increase in the amount of glutamate loaded into synaptic vesicles. Sample traces from control and overexpressing larva are shown in Figure 4, $c$ and $d$. We observed an $\sim 60 \%$ increase in mean mEJP amplitude, from $0.98 \pm 0.07 \mathrm{mV}$ in controls to $1.59 \pm$ 

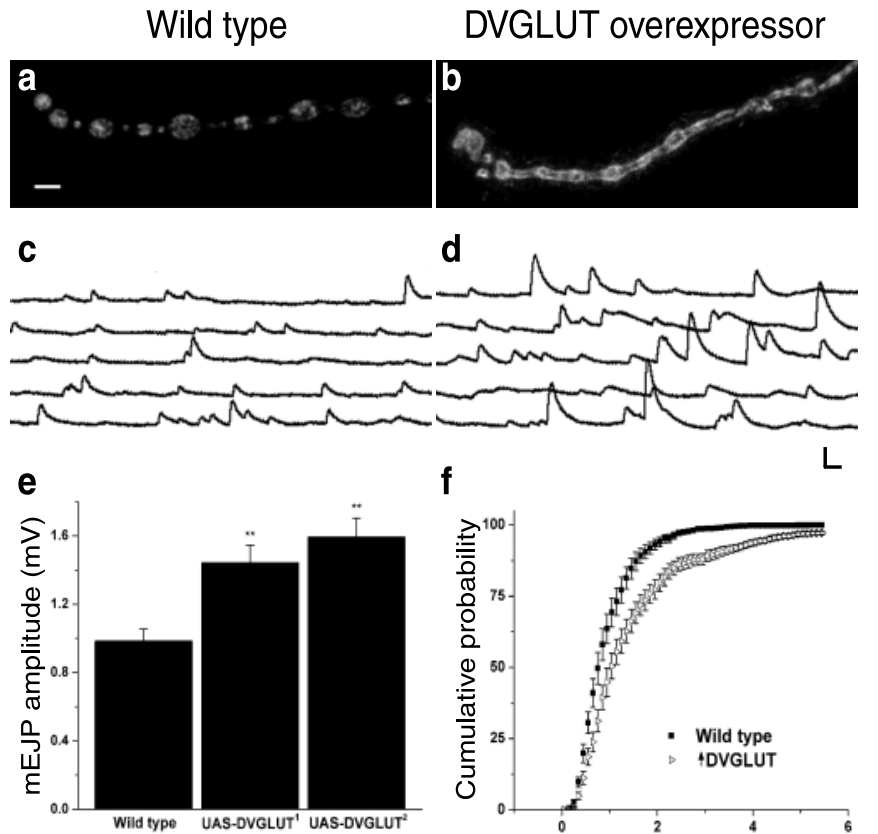

f

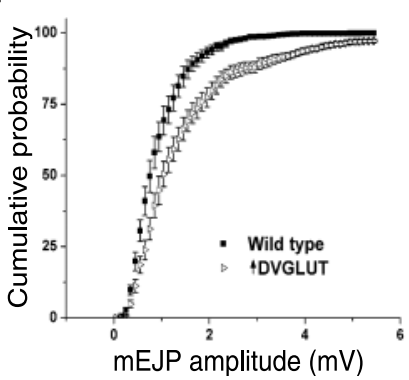

Figure 4. DVGLUT regulates quantal size. $a, b$, Representative confocal projections of synapses on muscle 4 in wild-type (BG380 Gal4/+) (a) and DVGLUT-overexpressing (BG380 Gal4/+;UAS-9887 $2 /+$ ) (b) larvae stained with anti-DVGLUT. Synaptic DVGLUT immunoreactivity is increased approximately threefold with overexpression. $c, d$, Representative spontaneous mEJPs recorded from muscle 6 of wild-type $(c)$ and DVGLUT-overexpressing $(d)$ larvae in nominally $\mathrm{Ca}^{2+}$-free saline containing $1 \mu \mathrm{M}$ TTX.e, Histogram showing the mean \pm SEM of spontaneous $\mathrm{mEJP}$ amplitude of wild-type and DVGLUT-overexpressing larvae. Results from two independent UAS-DVGLUT transgenes (UAS-9887 ${ }^{1}$ and UAS-9887 ${ }^{2}$ ) are shown $\left({ }^{* *} p<\right.$ 0.002).f, Cumulative probability histogram of mEJP amplitudes from wild-type (closed squares) and DVGLUT-overexpressing (BG380 Gal4/+ ;UAS-9887²/+; open triangles) larvae. Each data point represents the average cumulative probability \pm SEM $(n=12$ cells). The population of events in the overexpressor is shifted toward larger amplitude events compared with the wildtype population. Calibration: $2 \mathrm{mV}, 200 \mathrm{msec}$.

$0.1 \mathrm{mV}$ in overexpressing larvae $(p<0.001 ; n=12)$. Independent insertions of the UAS-DVGLUT transgene show significant increases in mean quantal size (Fig. 4e), so this phenotype is caused by the presence of the transgene and not its site of insertion in the genome. The change in quantal size requires the expression of DVGLUT, because UAS-DVGLUT transgenes in the absence of GAL-4 had no significant effect on mean quantal size ( $p>0.2 ; n=10$; data not shown).

This increase in quantal size could be caused by the addition of a few very large events or a shift in the size of all events. To investigate these two possibilities, we constructed cumulative probability histograms of mEJP amplitudes from control and DVGLUT-overexpressing larvae (Fig. $4 f$ ). These two distributions are significantly different by the Kolmogorov-Smirnov test ( $\mathrm{p} \ll 0.001)$. The cumulative probability shown in Figure $4 d$ is an average cumulative probability \pm SEM from 12 cells for each genotype. Beginning at the third bin $(0.25 \mathrm{mV})$, the two cumulative probabilities are significantly different in each bin by the $t$ test $(p<0.05)$. This analysis suggests that the entire population of events is increased in size, with the median increasing by $35 \%$. In fact, when we scale the control mEJP distribution by 1.35 and compare it with the distribution from overexpressing larvae, these distributions overlap almost completely and are not statistically different (Kolmogorov-Smirnov test; $p=0.09$ ).

These data strongly suggest that the increase in mean quantal size is driven at least in part by the $35 \%$ increase in individual
mEJPs; however, we have also observed that $~ 1.4 \%$ of mEJPs are $>8 \mathrm{mV}$ and occasional mEJPs are $>20 \mathrm{mV}$. These large events skew the mean quantal size, thus resulting in the $60 \%$ mean increase but only a $35 \%$ median increase. We find that these occasional large $(>8 \mathrm{mV})$ events persist in the presence of TTX and in the absence of external calcium; therefore, they do not represent evoked synaptic events that occur in response to residual nerve activity and calcium-dependent exocytosis. To investigate whether these events might contribute to evoked synaptic transmission, however, we performed a failure analysis. At low external $\mathrm{Ca}^{2+}$ concentrations, nerve stimulation usually results in the fusion of either one vesicle (an event) or no vesicles (a failure). We used a calcium concentration $(0.23 \mathrm{~mm})$ that resulted in $\sim 50 \%$ failures in mutant larvae to bias events toward low numbers of vesicles released in response to a stimulus. We evoked $>3000$ events but did not observe any evoked event $>8 \mathrm{mV}$ in larvae overexpressing DVGLUT, in contrast to the relatively infrequent but consistently observed large spontaneous mEJPs; the $1.4 \%$ of mEJPs $>8 \mathrm{mV}$ represent 12 of 840 events. We conclude that the large events are not evocable by an action potential. As such, the median increase in quantal size of $35 \%$ more accurately describes the functionally relevant increase in quantal size that occurs when DVGLUT is overexpressed. Together, these data demonstrate that overexpression of DVGLUT leads to (1) an increase in the amplitude of the entire population of quantal events and (2) the generation of a small population of very large events that are not evocable. The demonstration that DVGLUT overexpression shifts the entire distribution of mEJPs to larger amplitudes supports recent similar findings with mouse VGLUT1 (Wojcik et al., 2004).

In addition to the measured increase in $\mathrm{mEJP}$ amplitude, we also observed a small increase in $\mathrm{mEJP}$ frequency in both genotypes that overexpress DVGLUT $(\mathrm{BG} 380 \mathrm{Gal} 4 /+=2.0 \pm 0.3 \mathrm{~Hz}$; BG380 Gal4/+; UAS-DVGLUT $/+=4.1 \pm 0.4 \mathrm{~Hz} ; \mathrm{BG} 380$ Gal4/+; UAS-DVGLUT ${ }^{2} /+=2.8 \pm 0.2 \mathrm{~Hz} ;(n=12,16,12 ; p<$ $0.05)$. This apparent increase in mEJP frequency suggests that in wild type the smallest events may be lost in the noise of the recordings. Hence, the measured increase in mEJP amplitude may underestimate the true increase in quantal size, because these smallest events are being measured in the DVGLUT overexpressors but not in wild type.

\section{DVGLUT expression increases SV volume}

The increase in quantal size with DVGLUT overexpression indicates that more glutamate is loaded in each vesicle. This may occur by increasing the concentration of neurotransmitter in the vesicle or by increasing the volume of the vesicle. For the storage of neuromodulatory monoamine neurotransmitters in specialized secretory granules, an increase in vesicular loading is coupled with an increase in vesicle volume (Colliver et al., 2000; Pothos et al., 2002; Gong et al., 2003). In contrast to monoamines, fast transmitters such as glutamate are stored exclusively in synaptic vesicles, and the relationship between transporter expression and synaptic vesicle volume has not been explored.

To investigate this issue using overexpression of DVGLUT, we performed an ultrastructural analysis of synaptic vesicle size at the Drosophila NMJ. We reasoned that if transmitter concentration remains constant, the increase in quantal size observed using electrophysiological methods should correspond morphologically to an increase in vesicle volume. Alternatively, if the concentration of transmitter within a vesicle represents a steady-state value that can be changed by increased transporter activity, a change in vesicle size would not be expected. To distinguish be- 

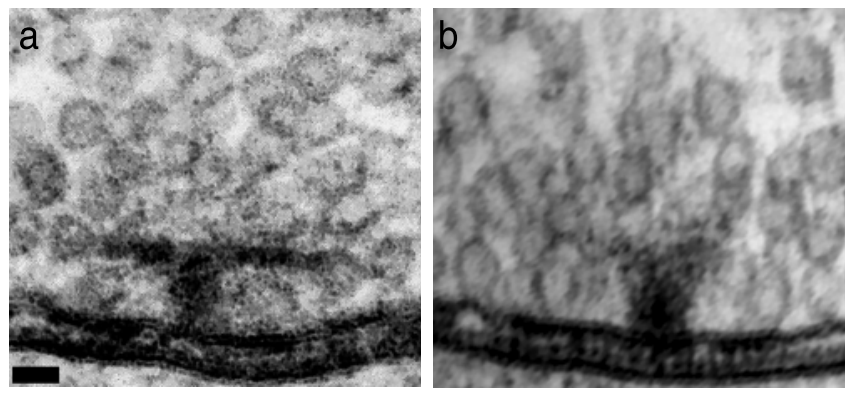

C

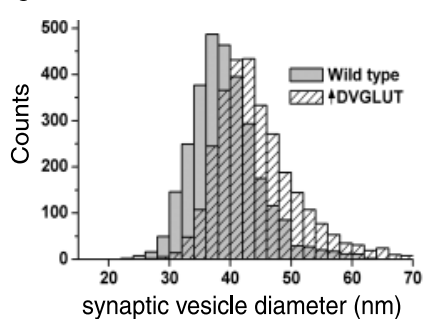

d

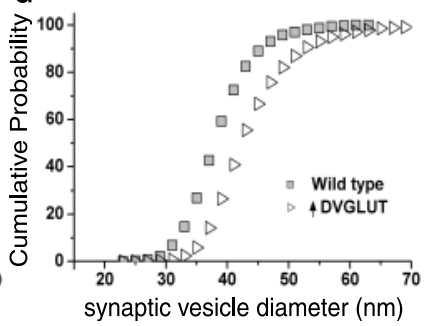

Figure 5. DVGLUT regulates synaptic vesicle size. $a, b$, Representative electron micrographs of active zones surrounded by a halo of synaptic vesicles from the type Ib NMJ onto muscle 6 from wild-type (BG380 Gal4/+) (a) and DVGLUT-overexpressing (BG380 Gal4/+; UAS$9887^{2} /+$ ) larvae (b). Scale bar, $50 \mathrm{~nm}$. c, Frequency histogram of measured synaptic vesicle outer diameter from wild-type (BG380 Gal4/+ ; gray bars) and DVGLUT overexpressing (BG380 Gal4/+; UAS- $9887^{2} /+$; hatched bars) larvae. $d$, Cumulative probability histogram of measured synaptic vesicle outer diameter from wild-type (gray squares) and DVGLUToverexpressing (open triangles) larvae. The entire population of events in the overexpressor is shifted toward larger diameters compared with the wild-type population.

tween these two potential mechanisms of increasing glutamate storage and release, we measured the size of 1700 synaptic vesicles at the NMJ of the type Ib motoneuron that innervates muscles 6 and 7 from both wild-type larvae and larvae overexpressing DVGLUT (Fig. $5 a, b$ ). We found a $14 \%$ increase in mean vesicle outer diameter, from $40.0 \pm 0.1 \mathrm{~nm}$ in controls to $45.6 \pm 0.1 \mathrm{~nm}$ in DVGLUT overexpressors, and a median increase in vesicle outer diameter of $10 \%(39.7 \pm 0.1 \mathrm{~nm}$ in controls to $43.5 \pm 0.1$ $\mathrm{nm}$ in DVGLUT overexpressors). We also measured SV membrane thickness and subtracted it from the outer diameter measurements to yield estimates of inner diameter, which are more directly proportional to volume. With an average membrane thickness of $8.7 \pm 0.1 \mathrm{~nm}$, estimated vesicle volume changes by $61 \%$ (median) or $95 \%$ (mean).

The increase in vesicle diameter and hence volume is similar to the increase in quantal size measured by electrophysiology. In both cases, the entire populations are shifted to the right relative to wild type, with the appearance of a few very large quanta and vesicles that skew the distribution more to the right (Fig. $5 c, d$ ). These results suggest that overexpression of DVGLUT increases quantal size by increasing the volume of synaptic vesicles without an increase in transmitter concentration.

\section{A homeostatic response to excess glutamate release}

The Drosophila GAL4/UAS system allows us to overexpress DVGLUT within its normal synaptic environment and thereby test its effect in vivo on an intact synapse. Having demonstrated that increased DVGLUT expression can regulate vesicular volume and quantal size, we wondered how this excess glutamate release might affect the efficacy of synaptic transmission. To assess synaptic function, we recorded evoked EJPs from muscle 6 of segments A3 and A4 in saline containing $0.47 \mathrm{mM} \mathrm{Ca}^{2+}$ (Fig. $6 a, b)$. Surprisingly, we found no significant difference in the am- a

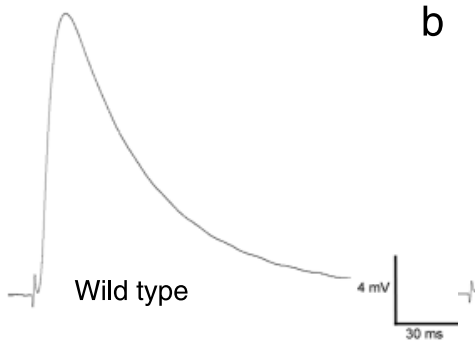

b
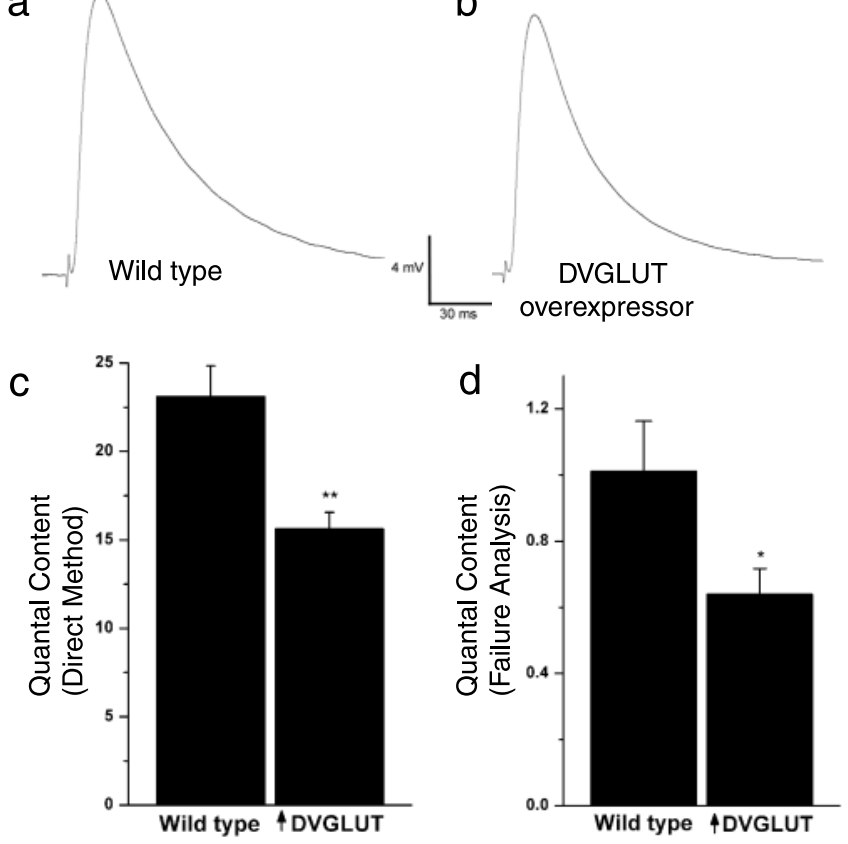

Figure 6. A decrease in quantal content maintains normal evoked release in DVGLUToverexpressing larvae. Representative evoked junctional potentials from wild-type (a) and DVGLUT-overexpressing ( $b$ ) larvae. Analysis of a number of cells ( $n=13$ for each genotype) indicates that the amplitude of evoked events is not significantly different. c, Histogram showing the mean \pm SEM of the quantal content for wild-type and DVGLUT-overexpressing larvae recorded in $0.47 \mathrm{~mm}$ calcium. Quantal content is calculated by the direct method (QC $=$ EJP/ $m E J P$, where the median mEJP is used; see Results). $d$, Histogram showing the mean \pm SEM of the quantal content for wild-type and DVGLUT-overexpressing larvae recorded in $0.23 \mathrm{~mm}$ calcium and calculated by the method of failures [QC $=\ln$ (trials/failures)]. Two independent methods of estimating quantal content indicate that the number of synaptic vesicles released from the DVGLUT overexpressors is decreased relative to wild type $\left({ }^{* *} p<0.01\right.$; ${ }^{*} p<0.05$ ).

plitude of evoked events (EJP: $17.4 \pm 1.0 \mathrm{mV}$ in controls and $16.0 \pm 1.0 \mathrm{mV}$ in larvae overexpressing DVGLUT; $n=13$ ), despite the increase in the amplitude of single mEJPs. Because the DVGLUT overexpressors show an increased response to a single vesicle but an apparently wild-type response to the evoked release of many vesicles, we hypothesized that fewer vesicles may be released in the overexpressor.

To assess whether fewer vesicles were released in the overexpressor we obtained an estimate of the QC. The simplest estimate of $\mathrm{QC}$ is given by the direct method ( $\mathrm{QC}=\mathrm{EJP} / \mathrm{mEJP})$, where $\mathrm{mEJP}$ represents the mean value of observed $\mathrm{mEJPs}$; however, the mean increase in the mEJP that we observed is likely to overestimate the increase in response to most individual vesicles, because the rare and very largest events are not evocable and skew the mean to the right. To minimize this potential error, we calculated quantal content using the median instead of mean mEJP. Using this variation, we found that quantal content decreased by $33 \%$, from $23.1 \pm 1.7$ in controls to $15.6 \pm 0.9$ in larvae overexpressing DVGLUT (Fig. $6 c)(n=13 ; p<0.01)$, strongly suggesting a decrease in vesicle release. To obtain a second estimate of QC that is independent of the observed size of the $\mathrm{mEJP}$, we also calculated quantal content using failure analysis. Failure analysis does not rely on the mEJP amplitude and does not assume that the same vesicles contribute to spontaneous and evoked events. Instead, this method of estimating vesicle release is based on the assumption that vesicular transmitter release will follow Poisson statistics when the probability of release approaches zero from a large number of release sites (Del Castillo and Katz, 1954). For 
this method, quantal content is estimated as the natural log of the ratio of trials of nerve stimulation to the number of failures of the nerve to release transmitter. Many previous studies demonstrate that these assumptions are valid at the Drosophila NMJ under conditions of low external calcium (Petersen et al., 1997; Davis and Goodman, 1998; Haghighi et al., 2003), and we therefore performed similar studies using $0.23 \mathrm{~mm}$ external $\mathrm{Ca}^{2+}$. Under these conditions, synapses overexpressing DVGLUT were more likely than wild-type synapses to fail to release a vesicle. Calculating by the method of failures demonstrated a $37 \%$ decrease in quantal content $(\mathrm{QC}=1.01 \pm 0.15$ in controls; $\mathrm{QC}=0.64 \pm$ 0.08 in overexpressing larvae; $n=12 ; p<0.05$ ) (Fig. $6 d$ ). Therefore, two independent estimates of quantal content demonstrate that when DVGLUT is overexpressed and more glutamate is released from a single vesicle, the synapse responds by releasing fewer vesicles. This suggests that homeostatic mechanisms at this synapse can decrease quantal content to maintain normal levels of synaptic excitation in response to excess glutamate release.

\section{Discussion}

We have investigated how the expression of a vesicular glutamate transporter regulates the strength of a glutamatergic synapse in vivo. Our goal was to perform these studies at the Drosophila neuromuscular junction, a well characterized glutamatergic synapse, and thereby avoid the use of cultured neurons. We therefore identified and characterized DVGLUT. We demonstrate that DVGLUT is a synaptic vesicle protein that is present in the synaptic terminals of all glutamatergic motoneurons as well as many synaptic terminals in the CNS. Increased expression of DVGLUT at the NMJ leads to an increase in quantal size. Hence, DVGLUT expression can regulate the glutamate content of a synaptic vesicle. We observe a corresponding increase in vesicle volume suggesting that the vesicular glutamate concentration is likely to remain roughly constant despite excess glutamate content. Release of this excess glutamate triggers a homeostatic mechanism that downregulates the number of vesicles released by the motoneuron, thereby maintaining normal levels of synaptic excitation.

\section{A Drosophila vesicular glutamate transporter}

We identified the predicted Drosophila protein CG9887 as a candidate DVGLUT because its amino acid sequence and hydrophobicity profile are similar to those of known VGLUTs and its mRNA is strongly expressed in a subset of neurons. We have demonstrated that CG9887 is a synaptic vesicle protein, is expressed at synaptic terminals of all known glutamatergic motoneurons, and can regulate the glutamate content of synaptic vesicles. Based on these findings, we conclude that CG9887 is a Drosophila VGLUT. Because CG9887 is the only Drosophila gene similar to the vertebrate VGLUTs that is expressed in neurons, CG9887 may be the only Drosophila VGLUT.

Although the Drosophila NMJ is a well characterized glutamatergic synapse, the role of glutamate as a transmitter elsewhere in the Drosophila nervous system has received little attention. We detect widespread expression of DVGLUT in the synaptic neuropil of the brain and nerve cord, suggesting that glutamate is an important transmitter for interneurons. The ability to detect glutamatergic terminals using the antibody to DVGLUT will aid in the identification of the transmitter phenotype of identified central neurons in Drosophila.

\section{DVGLUT regulates quantal size}

Overexpression of transporters for acetylcholine, monoamines, and glutamate in cultured cells can increase quantal size (Song et al., 1997; Colliver et al., 2000; Pothos et al., 2000; Wojcik et al., 2004), suggesting that changing the expression or activity of vesicular transporters may be a general mechanism for controlling the transmitter content of vesicles. We have now tested this hypothesis for a vesicular glutamate transporter at an intact synapse in vivo.

We generated transgenic flies that overexpress DVGLUT at the glutamatergic NMJ. Increased expression of DVGLUT led to a large increase in the postsynaptic response to the fusion of single vesicles. This increase in the mean response was attributable to two factors. First, the entire population of spontaneous events shifted to larger amplitude. The median amplitude increased by $35 \%$, and the mEJP amplitude distribution was recapitulated by scaling the wild-type distribution by a factor of 1.35 . Second, a small population of very large spontaneous events appeared with DVGLUT overexpression. These events persisted in the presence of TTX and absence of external calcium, so they do not represent spontaneous evoked release and could not be evoked by an action potential. Regardless of the nature of these rare events, the key finding is that increasing the levels of synaptic DVGLUT increases the postsynaptic response to the entire pool of synaptic vesicles. Hence, the number of DVGLUT molecules in a vesicle regulates the glutamate content of that vesicle.

Increased DVGLUT expression alters synaptic vesicle volume The concentration and volume of glutamate in the vesicle will affect the concentration and time course of glutamate in the synaptic cleft, which are important determinants of the postsynaptic response (Choi et al., 2000; Renger et al., 2001; Liu, 2003; Pawlu et al., 2004). The overexpression of DVGLUT loads more glutamate into vesicles, so either the concentration of glutamate or the size of the vesicle must have increased. To determine which mechanism is underlying the observed increase in quantal size, we measured the diameter of synaptic vesicles from wild-type and DVGLUT-overexpressing synapses. We observed that the entire population of synaptic vesicles is larger when DVGLUT is overexpressed. The calculated increases in the median (61\%) and mean $(95 \%)$ vesicle volume are more than sufficient to explain the observed increase in the median $(35 \%)$ and mean $(60 \%)$ quantal size. Therefore, it is likely that the glutamate concentration in the vesicle does not increase with DVGLUT overexpression; however, because quantal size does not change as much as vesicular volume, the glutamate concentration may actually be somewhat lower than in wild type. Alternatively, postsynaptic glutamate receptors may be approaching saturation in the mutant and unable to faithfully record the full extent of the increase in released glutamate, although the increase in quantal size that we observe demonstrates that in wild type the glutamate receptors are not saturated.

In previous studies of VGLUT and VAChT overexpression, the increases in quantal size were interpreted as increases in the concentration of vesicular transmitter (Song et al., 1997; Williams, 1997; Schuske and Jorgensen, 2004; Wojcik et al., 2004). This led to a steady-state model of vesicle filling, in which inflow through the transporters is balanced by a leak from the vesicle: more inflow caused by more transporter therefore leads to a higher steady-state equilibrium concentration of transmitter. This was contrasted with a set-point model, in which the concentration of transmitter is held constant, and more transporters would only be expected to increase the rate, but not the extent, of vesicle filling (Williams, 1997); however, these models do not take into account the possibility that the volume of the vesicle could change. Our data argue for a modified set-point model: the 
concentration of transmitter is held roughly constant, but the vesicular volume is changed by the number of transporter molecules in the vesicle. A similar model has been described for the loading of monoamines into secretory vesicles (Colliver et al., 2000; Gong et al., 2003). Our findings suggest that despite differences between secretory vesicles and synaptic vesicles, a similar mechanism can increase transmitter content.

Two models could explain the relationship between transporter expression and vesicle volume. First, the volume of the vesicle may be affected by its transmitter content; more functional transporter would add more glutamate, which by an unknown mechanism would lead to a larger vesicle. Alternatively, the vesicle volume may be sensitive to the physical addition of the transporter with its many membrane-spanning domains. These models could be distinguished by the expression of a nonfunctional transporter.

\section{A homeostatic response to excess glutamate release}

Having used transgenic techniques to manipulate DVGLUT expression in vivo, we were able to ask about the physiological consequences of this excess glutamate at an intact synapse. We find that total synaptic excitation is unchanged despite the increase in glutamate per vesicle because the presynaptic terminal releases fewer vesicles with each evoked event. It is possible that overexpression of DVGLUT directly decreases release probability; however, it would be an unlikely coincidence that this would exactly offset the increase in quantal size and produce a normal-sized evoked event. Instead, we favor the model that a modest and persistent increase in glutamate release from each vesicle leads to a compensatory decrease in the number of released vesicles that attenuates the excitatory effect of glutamate. Other homeostatic mechanisms have been described at the fly NMJ. Various studies have demonstrated that decreasing postsynaptic activity, either by manipulating glutamate receptors or postsynaptic potassium channels, triggers a compensatory increase in presynaptic transmitter release (Petersen et al., 1997; Davis et al., 1998; Paradis et al., 2001). In addition, nonvesicular leak of glutamate can homeostatically regulate postsynaptic glutamate receptor levels (Featherstone et al., 2002). The current findings are the first to demonstrate that a change in the release of vesicular glutamate can trigger a homeostatic change in presynaptic release properties.

What could trigger this compensatory downregulation of presynaptic release? Is it caused by increased postsynaptic excitation, or is it a direct effect of the increased glutamate in the cleft? We have previously manipulated postsynaptic expression of glutamate receptors leading to an increase in quantal size that is commensurate with the increase seen with DVGLUT overexpression. With increased postsynaptic excitation but no change in extracellular glutamate, there is no compensation; quantal content is unchanged and evoked synaptic events are larger (Petersen et al., 1997). So increasing glutamate release by overexpressing DVGLUT induces compensation, whereas directly increasing postsynaptic excitation by manipulating glutamate receptors does not affect cleft glutamate and does not trigger a compensatory decrease in quantal content. In summary, postsynaptic reductions in quantal size do initiate homeostatic compensation and postsynaptic increases in quantal size do not cause such compensation, but presynaptic increases in quantal size do trigger homeostatic decreases in presynaptic release. These data are consistent with the model that increased glutamate in the cleft directly triggers a compensatory downregulation of release. Similar findings in culture demonstrate that the persistent excitation of vertebrate neurons leads to a compensatory decrease in gluta- mate release (Moulder et al., 2004). Such homeostatic mechanisms could serve to limit the spread of excitotoxicity.

\section{References}

Bellocchio EE, Reimer RJ, Fremeau Jr RT, Edwards RH (2000) Uptake of glutamate into synaptic vesicles by an inorganic phosphate transporter. Science 289:957-960.

Brand AH, Perrimon N (1993) Targeted gene expression as a means of altering cell fates and generating dominant phenotypes. Development 118:401-415.

Budnik V, Koh YH, Guan B, Hartmann B, Hough C, Woods D, Gorczyca M (1996) Regulation of synapse structure and function by the Drosophila tumor suppressor gene dlg. Neuron 17:627-640.

Choi S, Klingauf J, Tsien RW (2000) Postfusional regulation of cleft glutamate concentration during LTP at "silent synapses". Nat Neurosci 3:330-336.

Clift-O'Grady LLA, Lowe AW, Grote E, Kelly RB (1990) Biogenesis of synaptic vesicle-like structures in a pheochromocytoma cell line PC-12. J Cell Biol 110:1693-1703.

Colliver TL, Pyott SJ, Achalabun M, Ewing AG (2000) VMAT-mediated changes in quantal size and vesicular volume. J Neurosci 20:5276-5282.

Davis GW, Goodman CS (1998) Synapse-specific control of synaptic efficacy at the terminals of a single neuron. Nature 392:82-86.

Davis GW, DiAntonio A, Petersen SA, Goodman CS (1998) Postsynaptic PKA controls quantal size and reveals a retrograde signal that regulates presynaptic transmitter release in Drosophila. Neuron 20:305-315.

Del Castillo J, Katz B (1954) Quantal components of the end-plate potential. J Physiol (Lond) 124:560-573.

DiAntonio A, Burgess RW, Chin AC, Deitcher DL, Scheller RH, Schwarz TL (1993) Identification and characterization of Drosophila genes for synaptic vesicle proteins. J Neurosci 13:4924-4935.

Erickson JD, Eiden LE, Hoffman BJ (1992) Expression cloning of a reserpine-sensitive vesicular monoamine transporter. Proc Natl Acad Sci USA 89:10993-10997.

Erickson JD, Varoqui H, Schafer MK, Modi W, Diebler MF, Weihe E, Rand J, Eiden LE, Bonner TI, Usdin TB (1994) Functional identification of a vesicular acetylcholine transporter and its expression from a "cholinergic" gene locus. J Biol Chem 269:21929-21932.

Featherstone DE, Rushton E, Broadie K (2002) Developmental regulation of glutamate receptor field size by nonvesicular glutamate release. Nat Neurosci 5:141-146.

Fremeau Jr RT, Burman J, Qureshi T, Tran CH, Proctor J, Johnson J, Zhang H, Sulzer D, Copenhagen DR, Storm-Mathisen J, Reimer RJ, Chaudhry FA, Edwards RH (2002) The identification of vesicular glutamate transporter 3 suggests novel modes of signaling by glutamate. Proc Natl Acad Sci USA 99:14488-14493.

Fremeau Jr RT, Kam K, Qureshi T, Johnson J, Copenhagen DR, StormMathisen J, Chaudhry FA, Nicoll RA, Edwards RH (2004) Vesicular glutamate transporters 1 and 2 target to functionally distinct synaptic release sites. Science 304:1815-1819.

Gong LW, Hafez I, Alvarez de Toledo G, Lindau M (2003) Secretory vesicles membrane area is regulated in tandem with quantal size in chromaffin cells. J Neurosci 23:7917-7921.

Haghighi AP, McCabe BD, Fetter RD, Palmer JE, Hom S, Goodman CS (2003) Retrograde control of synaptic transmission by postsynaptic CaMKII at the Drosophila neuromuscular junction. Neuron 39:255-267.

Johansen J, Halpern ME, Johansen KM, Keshishian H (1989) Stereotypic morphology of glutamatergic synapses on identified muscle cells of Drosophila larvae. J Neurosci 9:710-725.

Karunanithi S, Marin L, Wong K, Atwood HL (2002) Quantal size and variation determined by vesicle size in normal and mutant Drosophila glutamatergic synapses. J Neurosci 22:10267-10276.

Kopczynski CC, Davis GW, Goodman CS (1996) A neural tetraspanin, encoded by late bloomer, that facilitates synapse formation. Science 271:1867-1870.

Liu G (2003) Presynaptic control of quantal size: kinetic mechanisms and implications for synaptic transmission and plasticity. Curr Opin Neurobiol 13:324-331.

Liu Y, Peter D, Roghani A, Schuldiner S, Prive GG, Eisenberg D, Brecha N, Edwards RH (1992) A cDNA that suppresses MPP+ toxicity encodes a vesicular amine transporter. Cell 70:539-551. 
Malinow R, Malenka RC (2002) AMPA receptor trafficking and synaptic plasticity. Annu Rev Neurosci 25:103-126.

Marrus SB, DiAntonio A (2004) Preferential localization of glutamate receptors opposite sites of high presynaptic release. Curr Biol 14:924-931.

Marrus SB, Portman SL, Allen MJ, Moffat KG, DiAntonio A (2004) Differential localization of glutamate receptor subunits at the Drosophila neuromuscular junction. J Neurosci 24:1406-1415.

McIntire SL, Reimer RJ, Schuske K, Edwards RH, Jorgensen EM (1997) Identification and characterization of the vesicular GABA transporter. Nature 389:870-876.

Moulder KL, Meeks JP, Shute AA, Hamilton CK, de Erausquin G, Mennerick S (2004) Plastic elimination of functional glutamate release sites by depolarization. Neuron 42:423-435.

Paradis S, Sweeney ST, Davis GW (2001) Homeostatic control of presynaptic release is triggered by postsynaptic membrane depolarization. Neuron 30:737-749.

Pawlu C, DiAntonio A, Heckmann M (2004) Postfusional control of quantal current shape. Neuron 42:607-618.

Petersen SA, Fetter RD, Noordermeer JN, Goodman CS, DiAntonio A (1997) Genetic analysis of glutamate receptors in Drosophila reveals a retrograde signal regulating presynaptic transmitter release. Neuron 19:1237-1248.

Pothos EN, Larsen KE, Krantz DE, Liu Y, Haycock JW, Setlik W, Gershon MD, Edwards RH, Sulzer D (2000) Synaptic vesicle transporter expression regulates vesicle phenotype and quantal size. J Neurosci 20:7297-7306.

Pothos EN, Mosharov E, Liu KP, Setlik W, Haburcak M, Baldini G, Gershon MD, Tamir H, Sulzer D (2002) Stimulation-dependent regulation of the $\mathrm{pH}$, volume and quantal size of bovine and rodent secretory vesicles. J Physiol (Lond) 542:453-476.

Renger JJ, Egles C, Liu G (2001) A developmental switch in neurotransmit- ter flux enhances synaptic efficacy by affecting AMPA receptor activation. Neuron 29:469-484.

Roghani A, Feldman J, Kohan SA, Shirzadi A, Gundersen CB, Brecha N, Edwards RH (1994) Molecular cloning of a putative vesicular transporter for acetylcholine. Proc Natl Acad Sci USA 91:10620-10624.

Schuske K, Jorgensen EM (2004) Neuroscience. Vesicular glutamate transporter-shooting blanks. Science 304:1750-1752.

Song H-J, Ming G-L, Fon E, Bellocchio E, Edwards RH, Poo M-M (1997) Expression of a putative vesicular acetylcholine transporter facilitates quantal transmitter packaging. Neuron 18:815-826.

Stewart BA, Atwood HL, Renger JJ, Wang J, Wu CF (1994) Improved stability of Drosophila larval neuromuscular preparations in haemolymphlike physiological solutions. J Comp Physiol [A] 175:179-191.

Takamori S, Rhee JS, Rosenmund C, Jahn R (2000) Identification of a vesicular glutamate transporter that defines a glutamatergic phenotype in neurons. Nature 407:189-194.

Tautz D, Pfeifle C (1989) A non-radioactive in situ hybridization method for the localization of specific RNAs in Drosophila embryos reveals translational control of the segmentation gene hunchback. Chromosoma 98:81-85.

van de Goor J, Ramaswami M, Kelly R (1995) Redistribution of synaptic vesicles and their proteins in temperature-sensitive shibire(ts1) mutant Drosophila. Proc Natl Acad Sci USA 92:5739-5743.

Williams J (1997) How does a vesicle know it is full? Neuron 18:683-686.

Wojcik SM, Rhee JS, Herzog E, Sigler A, Jahn R, Takamori S, Brose N, Rosenmund C (2004) An essential role for vesicular glutamate transporter 1 (VGLUT1) in postnatal development and control of quantal size. Proc Natl Acad Sci USA 101:7158-7163.

Zinsmaier KE, Eberle KK, Buchner E, Walter N, Benzer S (1994) Paralysis and early death in cysteine string protein mutants of Drosophila. Science 263:977-980 